técnicos necesarios para su uso son una conexión a internet y un navegador web desde el que acceder al sitio. Fue desarrollada en 2017 por Frank Fischer (Higher School of Economics, Moscú) y Carsten Milling (Hashtable, Berlín), y actualmente se encuentra en su versión 2.0. Está basada en dos bibliotecas JavaScript: React ${ }^{6}$ para construir la interfaz de la herramienta, y Sigma ${ }^{7}$ para generar las gráficas de redes. Además, el código de la herramienta fue lanzado de forma abierta y se encuentra disponible en el repositorio de código colaborativo GitHub ${ }^{8}$, de forma que puede ser ejecutado en otro servidor o incluso clonado para utilizarlo de manera local en un ordenador.

Actualmente está alojada dentro del sitio web del Drama Corpora Project (DraCor)9, aunque fue desarrollada antes que este. DraCor es un proyecto del Centre for Digital Humanities at HSE (Moscú, Rusia) y de la Universität Potsdam (Polonia), y ofrece corpus teatrales en distintas lenguas: ruso, latín, griego antiguo, sueco, castellano, alemán, italiano, tártaro y alsaciano; y de dos autores específicos: Shakespeare y Calderón de la Barca. En el sitio de este gran proyecto encontraremos el texto de un total de más de mil cien obras, con versiones descargables del texto hablado en formato TXT y JSON, y de las acotaciones en TXT. Además, están disponibles gráficas de redes y análisis estadísticos de las relaciones entre los personajes de cada obra, con los datos para generar la red descargables en formato CSV10, GEXF y GraphML. Tanto los archivos descargables con los datos para las redes, como los grafos que encontramos de todas las obras de este gran corpus, han sido generados con Easy Linavis. $Y$ es que gracias a esta herramienta podemos tanto generar redes como descargar un archivo csv con toda la información necesaria para importar y trabajar la red en otras herramientas.

Según los desarrolladores, Easy Linavis está diseñada principalmente para ser usada con fines didácticos, por lo que su propósito no es competir con otras herramientas de extracción de redes de datos de textos literarios (Fischer \& Milling, 2017, p. 175). Por otro lado, aunque se trata de una herramienta sencilla, es posible manejar una gran cantidad de datos y podría ser usada tanto para el análisis de personajes de obras dramáticas como de otro tipo de textos literarios. Además, la posibilidad de descargar en formato CSV los datos, amplía aún más las posibilidades que en principio nos brinda la herramienta.

Es posible acceder a este recurso web directamente usando la url https:// ezlinavis.dracor.org/, desde el enlace situado en la esquina superior derecha del sitio de DraCor,

\footnotetext{
1 Accesible desde: https://gephi.org/.

2 Accesible desde: https://cytoscape.org/.

3 Accesible desde: https://hdlab.stanford.edu/palladio/.

4 Ejemplo de ello son trabajos como Sparavigna (2014) o Rochat (2014).

5 Accesible desde: https://ezlinavis.dracor.org/.

6 React es una biblioteca JavaScript de código abierto diseñada para crear interfaces de usuario con el objetivo de facilitar el desarrollo de aplicaciones en una sola página (SPA). Accesible desde: https:// reactis.org/.

7 Sigma es una biblioteca JavaScript dedicada al dibujo de gráficas. Facilita la publicación de gráficos de redes en páginas web, y permite a los desarrolladores integrar la exploración de redes en aplicaciones web. Accesible desde: http://sigmajs.org/.

8 Accesible desde: https://github.com/dracor-org/ezlinavis.

9 Accesible desde: https://dracor.org/.

10 Del inglés: Comma-Separated Values (valores separados por comas). En este formato tabular cada coma ("") separa una columna de otra y cada salto de línea representa una fila.
} 
- simplemente escribiendo ezlinavis en nuestro buscador web, pues aparecerá rápidamente como el primer resultado de búsqueda.

La interfaz de Easy Linavis está dividida en tres columnas, y se puede explicar el uso de la herramienta de manera sencilla a través de ellas.

En la primera columna, introducimos los datos; en la segunda columna, estos datos aparecerán transformados a formato tabular; y en la tercera columna, se generará la visualización de la red.

Para introducir los datos, debemos utilizar un sencillo lenguaje de etiquetado: mediante el uso de asteriscos (\#) generamos la estructura jerarquizada del texto, y dentro de cada parte 0 segmento que creemos, introducimos los nombres de los personajes que aparezcan o hablen (según el criterio que estemos utilizando). Antes del primer asterisco, podemos escribir libremente metadatos sobre el texto, pues Easy Linavis no los tomará en cuenta. Dejo un ejemplo visual para aclarar esta cuestión:

\begin{tabular}{l|l}
$\begin{array}{l}\text { Titulo, } \\
\text { autor, } \\
\text { año, }\end{array}$ & $\begin{array}{l}\text { Titulo, } \\
\text { autor, } \\
\text { año, }\end{array}$ \\
.. & $\ldots$ \\
\# Acto 1 & \# Acto 1 \\
\#\# Escena 1 & \#\# Escena 1 \\
Personaje1 & Personaje1 \\
Personaje2 & Personaje2 \\
Personaje3 & Personaje3 \\
\#\# Escena 2 & \#\# Escena 2 \\
Personaje 1 & Personaje 1 \\
Personaje3 & Personaje3 \\
\# Acto 2 & \# Acto 2 \\
\#\# Escena 1 & \#\# Escena 1 \\
... & $\ldots$ \\
$\ldots$. & $\ldots$ \\
... & $\ldots$
\end{tabular}

Figura 1. Capturas de pantalla en las que se muestra el sistema de etiquetado para introducir los datos. Una franja de color en el lateral izquierdo nos indica si los datos se han introducido de manera correcta (izquierda, línea verde) o si existe algún error (derecha, línea roja). Fuente: elaboración propia.

En la herramienta no podemos importar nuestros datos desde ningún tipo de archivo externo, estos deben ser introducidos manualmente en el sitio web, o escritos en cualquier editor de texto para después realizar un simple copy/paste. Esto es debido a que una de las principales funcionalidades de Easy Linavis es facilitar la tarea de extracción de los datos sobre las relaciones de los personajes de un texto, mediante la utilización del etiquetado descrito. Además, es gracias a este sistema de inserción de datos que, por el código que incorpora, es capaz de transformar a formato tabular de 4 columnas toda la información necesaria para la composición del grafo.

Los datos que aparecerán automáticamente en la segunda columna de la interfaz de la herramienta se encuentran en formato CSV, y están disponibles para descargar haciendo clic en download csv: 


\section{download CSV}

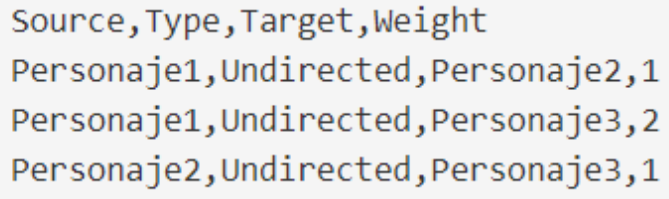

Figura 2. Captura de pantalla de la segunda columna, en donde vemos la tabla con los datos en formato csv y la opción de descarga del archivo generado. Fuente: elaboración propia.

Este archivo contiene los datos para las columnas source (nodo de origen), target (nodo de destino), weight (peso de la arista, es decir, número de veces que se relacionan source y target) y type (tipo de relación). Easy Linavis genera por defecto redes no-dirigidas, por lo que añade en la columna type la información undirected para todas las aristas.

Con esta esta información, y gracias a la biblioteca JS Sigma, Easy Linavis presenta una visualización de la red, en la que el tamaño de los nodos y las aristas es proporcional a su grado (información que se encuentra en weight). Esta red se genera de forma predeterminada utilizando el algoritmo de distribución (layout) Forcelink, pero esto puede modificarse en el menú desplegable que aparece al clicar sobre Graph, en la cabecera del sitio. En este menú tenemos disponibles otras opciones de algoritmos de distribución: NOverlap y ForceAtlas2, además de ForceLink.

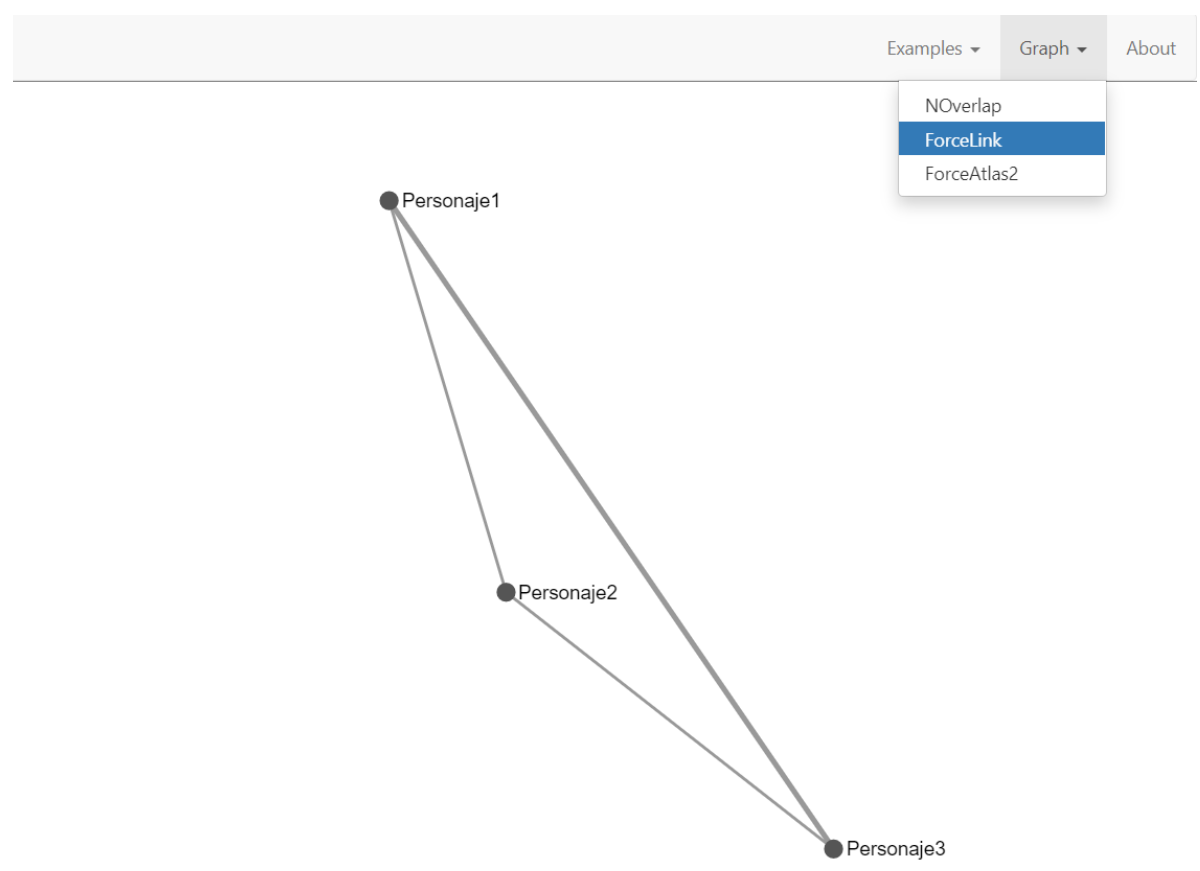

Figura 3. Captura de pantalla de la tercera columna y del menú en donde podemos seleccionar qué algoritmo de distribución queremos utilizar para el grafo. Fuente: elaboración propia.

Situándonos sobre la red, es posible aumentar y disminuir su tamaño utilizando el scroll (tanto en un ratón como en un touchpad o trackpad), o desplazarla haciendo clic izquierdo y arrastrando. Además, al pasar con el cursor sobre cualquier nodo, su etiqueta (en este caso, nombre del personaje) se resaltará mediante un fondo blanco bajo las letras; lo que es muy útil en una red con 
muchos nodos o muchas aristas en la que leer la etiqueta de un nodo puede ser complicado.

En la cabecera del sitio, encontramos un menú con otros dos ítems además del desplegable Graph ya descrito. En la primera opción, Examples, tenemos ejemplos de textos dramáticos entre los que podremos elegir para visualizar su red e incluso modificar sus datos o descargar el archivo CSV. Y si hacemos clic sobre About, el ítem del menú más a la derecha, se abrirá una ventana emergente con información sobre Easy Linavis y una breve explicación de uso.

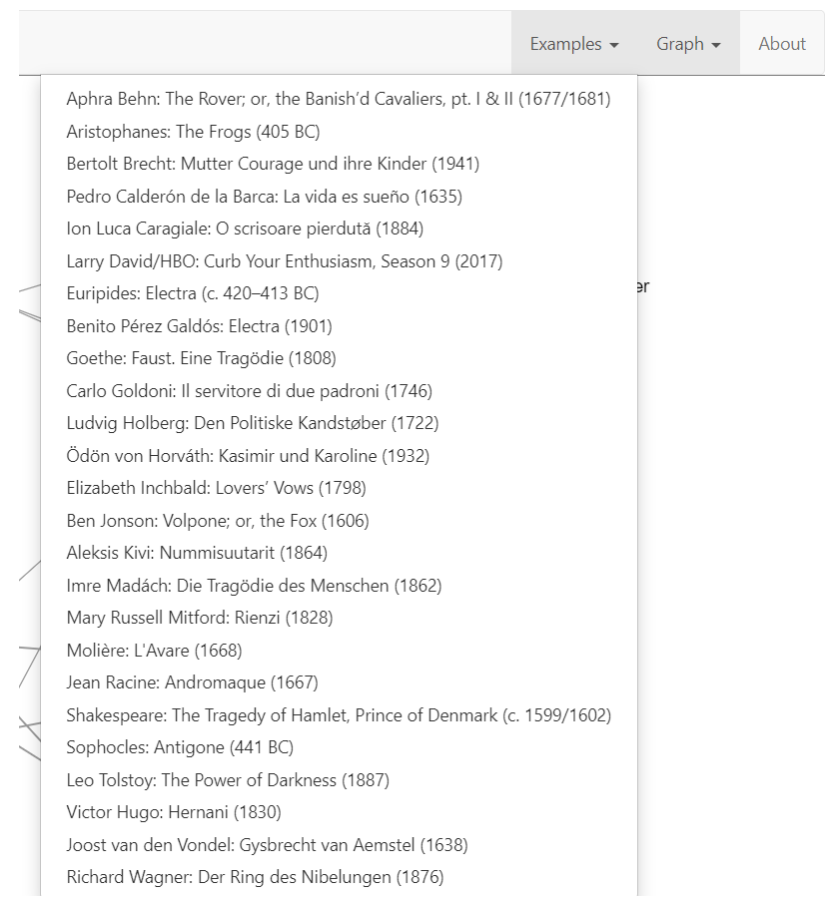

Figura 4. Captura de pantalla del menú desplegable Examples. Podemos aquí ver las veinticinco obras que Ezlinavis nos ofrece como ejemplo. Fuente: elaboración propia.

Por último, es importante tener en cuenta que Easy Linavis no almacena en ningún momento los datos que introducimos. Es decir, si cerramos la herramienta, los datos que hayamos introducido, así como el archivo CSV y la red, desaparecerán. Por lo tanto, es importante que, si trabajamos con muchos datos, tengamos esto en cuenta para ir guardando los datos que estemos ingresando en la herramienta, por ejemplo, en un documento de texto aparte, y así asegurarnos de que no perderemos la información en el caso de que ocurriese algún problema, como el cierre por error del navegador web o cualquier otra cuestión técnica.

Es posible que si nos centramos en el modelo de red que genera Easy Linavis pensemos que se trata de una herramienta simple o poco completa, pero creo que esta visión deja fuera gran parte de las funcionalidades de este recurso, pues quizá, generar redes no es en sí la principal funcionalidad de esta herramienta, sino un elemento algo más secundario.

Empezando por la introducción de los datos, Easy Linavis nos entrega un sencillo sistema de etiquetado, que considero fácil de comprender y utilizar para cualquier usuario sin conocimientos en lenguajes de programación; y gracias a él, conseguimos simplificar la compleja tarea de extracción de datos sobre personajes de un texto dramático. Gracias al algoritmo escrito por los desarrolladores, estos datos simplificados que introducimos se transforman automáticamente a formato tabular csv, tarea aún más complicada de realizar manualmente, y que con la herramienta 
se ve reducida a milésimas de segundo. Es este proceso de transformación de los datos la principal clave que hace de Easy Linavis un recurso tan potente para el trabajo en humanidades digitales y los estudios teatrales.

Este archivo csv descargable que se genera, nos abre las puertas a un mundo inmenso de opciones para trabajar con la red. Por ejemplo, es posible modificar este archivo con cualquier software de hojas de cálculo para añadir más información a la red con atributos de los nodos: el género de los personajes, información geográfica, o modificar el tipo de arista. Más tarde podremos utilizar estos nuevos datos en programas como Gephi o Cytoscape para mejorar la visualización de la red. O también, importar el archivo descargado directamente en estos softwares para realizar análisis cuantitativos de la red: modularidad, diámetro, centralidad, densidad, distancias entre nodos, etc. Las posibilidades para el análisis de redes se multiplican al disponer de los datos en este formato.

Es importante también tener en cuenta que la herramienta solo está disponible en inglés, pero gracias a herramientas como Google Translate podemos traducir al castellano u otro idioma el sitio web automáticamente, debido a su integración en navegadores basados en Chromium como Google Chrome o el nuevo Microsoft Edge. Esta traducción, aunque no perfecta, será suficiente en el caso de que quisiéramos utilizar este recurso pero supusiera una barrera idiomática que sólo esté disponible en inglés.

Por último, si entendemos Easy Linavis en la totalidad del proyecto, y no solo como una herramienta online, resulta de gran importancia considerar que ha sido desarrollado dentro del movimiento del código abierto. Como vimos, el código que utiliza la herramienta para funcionar, fue lanzado en GitHub, por lo que este puede ser utilizado de forma local en el ordenador, o lo que es más interesante: utilizarse en un servidor propio e integrarse en otros proyectos y recursos digitales.

Con todo lo descrito, creo necesario considerar Easy Linavis como una de las grandes herramientas digitales para humanistas e investigadores o docentes de literatura y teatro. Primero por el espectro de necesidades que logra cubrir con sus distintas y sencillas funcionalidades. $Y$, en segundo lugar, por estar pensado específicamente para el análisis de textos literarios, fomentando así el desarrollo de estudios teatrales a través de herramientas digitales y ampliando la cantidad de recursos de acceso libre disponibles.

\section{REFERENCIAS BIBLIOGRÁFICAS}

del Rio Riande, G. (2018). Reseña de: Know your Implementation: Subgraphs in Literary Networks. https://openmethods.dariah.eu/2018/02/12/know-your-implementation-subgraphs-inliterary-networks/

Fischer, F., Göbel, M., Kampkaspar, D., Kittel, C., \& Trilcke, P. (2017). Network Dynamics, Plot Analysis: Approaching the Progressive Structuration of Literary Texts. En DH2017. Book of Abstracts. McGill University. https://dh2017.adho.org/abstracts/071/071.pdf 
Fischer, F., Kittel, C., \& Trilcke, P. (2017). Know your Implementation: Subgraphs in Literary Networks. https://dlina.github.io/Subgraphs/

Fischer, F., \& Milling, C. (2017). Easy Linavis (Simple Network Visualisation for Literary Texts). En Rodríguez Ortega, N. (ed.), Libro de resúmenes del III Congreso de la Sociedad Internacional Humanidades Digitales Hispánicas: Sociedades, políticas, saberes (Málaga, 18-20 de octubre de 2017). Humanidades Digitales Hispánicas. Sociedad Internacional (HDH). https:// humanidadesdigitaleshispanicas.es/contribuciones/congreso-hdh-2017/

Flüh, M. (2019). Ezlinavis. En forTEXT. Literatur digital erforschen. https://fortext.net/tools/tools/ ezlinavis

Horstmann, J. (2019): DraCor: Drama Corpora Project. En: forTEXT. Literatur digital erforschen. https://fortext.net/ressourcen/textsammlungen/dracor-drama-corpora-project

Moretti, F. (201 1). Network Theory, Plot Analysis. Stanford Literary Lab Pamphlets, 2, 1-13. https:// litlab.stanford.edu/LiteraryLabPamphlet2.pdf

Moretti, F. (2013). Distant Reading. Verso.

Rochat, Y. (2014). Character Networks and Centrality. [Tesis doctoral]. Université de Lausanne. https://infoscience.epfl.ch/record/203889/files/yrochat thesis infoscience.pdf

Sparavigna, A. C. (2014). Graph Visualization Software for Networks of Characters in Plays. International Journal of Sciences, 3(02), 69-79. http://dx.doi.org/10.18483/iiSci.414 